\title{
Analysis of retinal and choroidal microvasculature in systemic sclerosis: an optical coherence tomography angiography study
}

\author{
Hilal Kılınç Hekimsoy ${ }^{1} \cdot$ Mehmet Ali Şekeroğlu $^{1}$ Ali Mert Koçer ${ }^{1} \cdot$ Ali Akdoğan ${ }^{2}$
}

Received: 20 May 2019 / Revised: 14 July 2019 / Accepted: 29 July 2019 / Published online: 25 September 2019

(c) The Author(s), under exclusive licence to The Royal College of Ophthalmologists 2019

\begin{abstract}
Background To investigate the retinal and choroidal microvasculature in patients with systemic sclerosis (SSc) who have no clinical evidence of retinopathy and to compare these structures with those of healthy control subjects.

Methods Forty-five patients with a confirmed diagnosis of SSc and no clinical evidence of retinopathy and 45 age- and sexmatched healthy control subjects were included in the study. After complete ophthalmological examination, all patients underwent optical coherence tomography angiography (OCTA) measurement (Optovue Inc., Fremont, California, USA) to assess the foveal avascular zone (FAZ) area; FAZ perimeter; acircularity index of FAZ; foveal density-300; superficial and deep capillary plexus vessel densities; and choriocapillaris flow area at 1,2, and $3 \mathrm{~mm}$ radius. The data from the right eyes of all participants were used for statistical analysis.

Results The superficial capillary plexus vessel density of the whole image, fovea, parafovea and perifovea, and the deep capillary plexus vessel density of the fovea were significantly lower than those in healthy control subjects $(p<0.05$ for all). No significant difference was found in the FAZ area, FAZ perimeter, acircularity index of FAZ, foveal density-300 and choriocapillaris flow area measurements between patients and controls $(p>0.05$ for all).

Conclusions We demonstrated the ability of OCTA to reveal early retinal microvascular alterations in patients with SSc without any clinical evidence of retinopathy. Reduced vessel density in SSc patients supports the hypothesis of widespread vascular injury, including ocular microcirculation.
\end{abstract}

\section{Introduction}

Systemic sclerosis ( $\mathrm{SSc}$ ) is a chronic multisystem disorder characterized by immune activation, widespread vasculopathy and generalized fibrosis with heterogeneous manifestations $[1,2]$. SSc is characterized by prominent vascular involvement that is not limited to the peripheral microcirculation of the skin but is also observed in the heart, lungs, kidneys, gastrointestinal tract and eyes. Involvement of the microvasculature is one of the earliest features of SSc and potentially contributes to progressive organ dysfunction via pathological changes, including disruption of the endothelium, mononuclear cell infiltration of the vessel

Hilal Kılınç Hekimsoy

hilal_kilinc_@hotmail.com

Ulucanlar Eye Training and Research Hospital, Ankara, Turkey

2 Department of Rheumatology, Hacettepe University Faculty of Medicine, Ankara, Turkey wall, and obliterative lesions. These structural vascular changes and prolonged vasospasm can cause inadequate blood circulation and may result in tissue damage, atrophy, and even infarction of the capillaries [3-5]. Many ocular manifestations have been described involving both anterior and posterior segments, such as eyelid skin fibrosis, keratoconjunctivitis sicca, anterior uveitis, normal tension glaucoma, optic neuropathy, primary retinopathy and choroidopathy [6-8]. Involvement of the posterior segment is often subclinical, and visual loss directly related to the disease is rarely observed $[7,8]$.

Optical coherence tomography angiography (OCTA) is a dye-free imaging technique that can detect angiographic features of the macular capillary network and blood flow characteristics [9]. Compared with fundus fluorescein angiography, OCTA has the following advantages: higher resolution, less time consuming and more noninvasive $[10,11]$.

Retinal and choroidal microvascular changes are important in SSc patients because it is practically a disease of the small arteries [3, 4]. Therefore, the retinal and choroidal 
vasculature would be ideal for the observation of generalized arteriolar and capillary pathology in SSc. The aim of this prospective cross-sectional study was to investigate the retinal and choroidal microvasculature in patients with SSc who have no clinical evidence of retinopathy and to compare these results with those of healthy control subjects.

\section{Patients and methods}

This prospective cross-sectional study was performed at the Ankara Ulucanlar Eye Training and Research Hospital. Patients with no clinical evidence of eye disease were consecutively recruited from the outpatient clinic of the Department of Rheumatology, Hacettepe University Faculty of Medicine, a tertiary care referral center for SSc. The study followed the tenets of the Declaration of Helsinki and was approved by the institutional review board. Informed written consent was obtained from each patient.

A total of 45 eyes of 45 consecutive patients with systemic SSc were enrolled in this study, and the data of these patients were compared to those of 45 age- and sex-matched controls who had no ocular or systemic diseases. Data regarding age, gender, SSc subtype, disease duration, age at diagnosis, autoantibody profile, and the type of systemic treatment were recorded. Patients with SSc sine scleroderma, scleroderma overlap syndrome, diabetes mellitus, severe systemic hypertension (defined as systolic blood pressure exceeding $179 \mathrm{mmHg}$ or a diastolic blood pressure exceeding $109 \mathrm{mmHg}$ ) and pulmonary hypertension were excluded. Exclusion criteria also encompassed the presence of other retinal diseases, severe cataract, nystagmus, poor eye fixation, significant media opacity and refractive spherical and cylindrical error $\geq 2 \mathrm{D}$ that could possibly confound the observation. Following detailed ophthalmologic examination including the Snellen best corrected visual acuity test, slit-lamp examination, intraocular pressure measurement and dilated indirect ophthalmoscopy, OCTA, which was not part of the standard clinical care, was performed in all participants with the XR Avanti AngioVue OCTA (Optovue, Fremont, California, USA) (Version 2017.1.0.151) at the same time interval (2-4 p.m.) of the day. This system uses a split-spectrum amplitude decorrelation angiography (SSADA) algorithm and operates at 70,000 A-scans per second to acquire OCTA volumes consisting of $400 \times 400 \mathrm{~B}$-scans [9-11]. Three-dimensional OCTA scans were acquired over $6 \mathrm{~mm} \times 6 \mathrm{~mm}$ regions, and all participants underwent three different types of scanning: cross-line scans, retina-map scans, and angio-retina scans. The angio-retina scans automatically inserted three foveacentered circles on the macula to detect the superficial capillary plexus (SCP) and deep capillary plexus (DCP) vessel densities and choriocapillaris flow area at 1,2 and 3 mm radius. Foveal zone vessel density was defined as the area of the small circle, with a diameter of $1 \mathrm{~mm}$. The parafoveal zone vessel density was defined as the area of the middle circle, with a diameter of $3 \mathrm{~mm}$. The perifoveal zone vessel density was defined as the area of the outer circle, with a diameter of $6 \mathrm{~mm}$. By using the software, the parafoveal and perifoveal regions were automatically divided into four fields: the superior, inferior, temporal, and nasal quadrants and two equal hemispheres (superior and inferior). The average vessel densities of four quadrants were calculated to analyze parafoveal and perifoveal values (Fig. 1). The foveal avascular zone (FAZ) area in the SCP was obtained via a nonflow assessment tool, and the FAZ area, including the full retinal vasculature, FAZ perimeter, acircularity index (AI) of the FAZ, and foveal density-300, were obtained via the FAZ assessment tool. The vascular flow area in which vascular signals were detected by the SSADA algorithm was drawn and calculated in a 3-mm radius circle in choriocapillaris. In addition, retinal and choroidal thicknesses were obtained from optical coherence tomography (OCT) images. The data from the right eyes of all participants were used for statistical analysis.

Statistical analyses were performed with Statistical Package for the Social Sciences (SPSS Inc., Chicago, Illinois, USA) version 20.0. Categorical variables were assessed by the Pearson Chi-square test or Fisher's exact test, as appropriate. To verify the normality of the data, we calculated normal probability plots and basic descriptive statistics (mean, standard deviation, min, and max) for every variable. Normality for continuous variables in each group was determined by the Kolmogorov-Smirnov test. Student's $t$ test was used for variables that showed a normal distribution. The Mann-Whitney $U$ test was used for variables that did not show a normal distribution. The correlations between the OCTA and OCT variables were investigated using Pearson correlation tests. For all tests, $p$ values less than 0.05 were considered statistically significant.

\section{Results}

Forty-five eyes of $45 \mathrm{SSc}$ patients with no clinical evidence of retinopathy and 45 eyes of 45 age- and sex-matched healthy control subjects were enrolled in this cross-sectional study. The mean duration of the disease was $10.51 \pm 8.20$ years (range 2-38 years) for SSc patients. There was no significant difference between the SSc and control groups in terms of age, gender distribution, spherical equivalent of refraction, and intraocular pressure $(p>0.05$ for all). The central macular thickness (CMT) did not significantly differ between SSc patient and healthy control eyes $(p=0.384)$, but the subfoveal choroidal thickness (SFCT) was significantly lower in SSc eyes $(p=0.001)$ on OCT. Table 1 


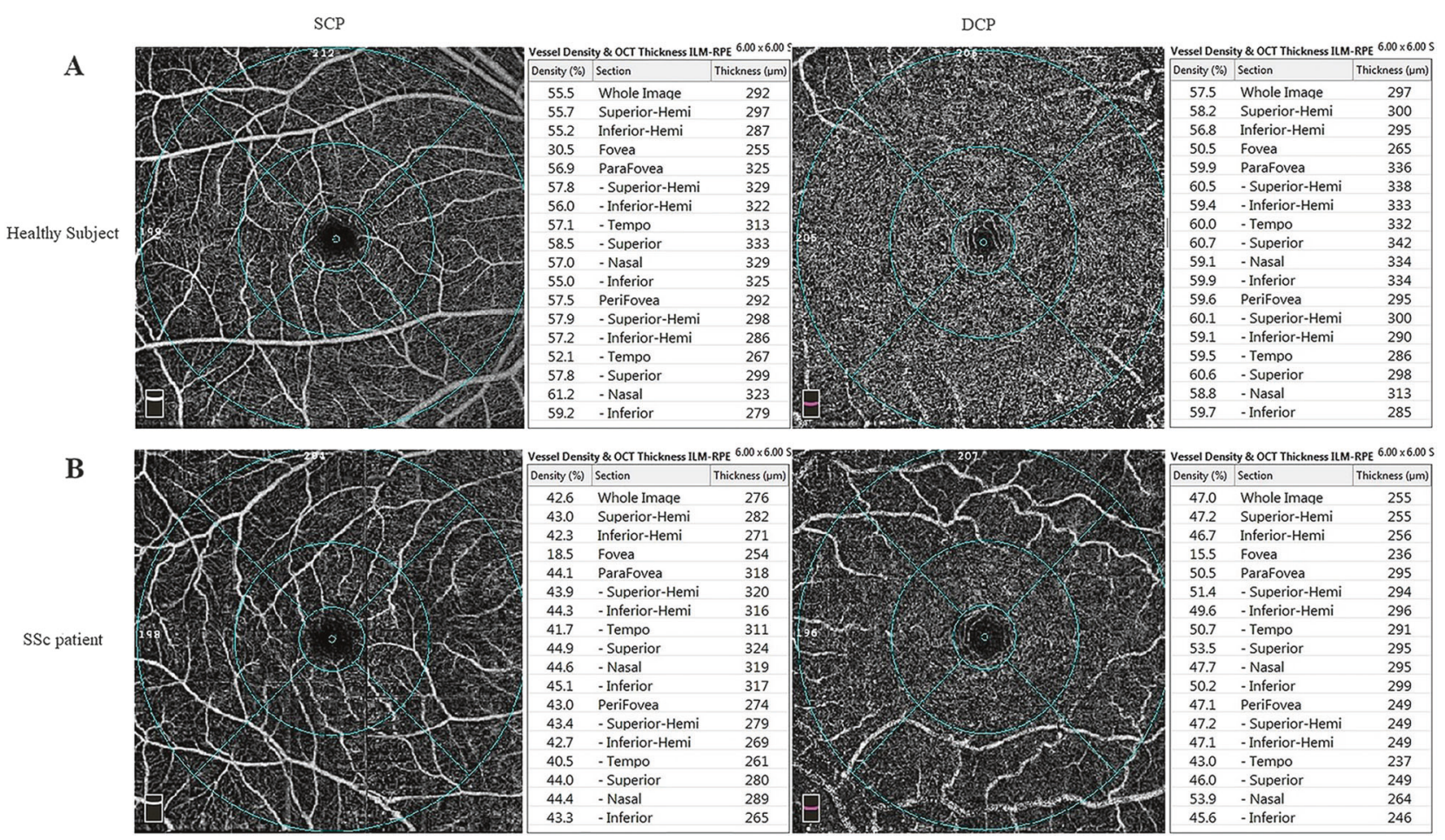

Fig. 1 Density assessment tool of optical coherence tomography angiography (OCTA) of the fovea in a healthy subject (a) and in a patient with systemic sclerosis (b)

Table 1 Clinical and demographic characteristics of SSc patients and control group

\begin{tabular}{|c|c|c|c|}
\hline Variable & SSc group & Control group & $p$ value \\
\hline \multicolumn{4}{|l|}{ Age (years) } \\
\hline Mean \pm SD & $52.00 \pm 8.20$ & $51.61 \pm 8.62$ & \multirow[t]{2}{*}{$0.668^{*}$} \\
\hline Range & $(26-73)$ & $(25-75)$ & \\
\hline \multicolumn{4}{|l|}{ Gender } \\
\hline Male (\%) & $3(6.66 \%)$ & $3(6.66 \%)$ & \multirow[t]{2}{*}{$0.852 * *$} \\
\hline Female $(\%)$ & $42(93.33 \%)$ & $42(93.33 \%)$ & \\
\hline \multicolumn{4}{|c|}{ Spherical equivalent, D } \\
\hline Mean \pm SD & $0.14 \pm 0.22$ & $0.41 \pm 0.65$ & \multirow[t]{2}{*}{$0.898 *$} \\
\hline Range & $(-0.5)-(+0,5)$ & $(-0.5)-(+1.0)$ & \\
\hline \multicolumn{4}{|l|}{ IOP, $\mathrm{mmHg}$} \\
\hline Mean \pm SD & $15.16 \pm 2.94$ & $15.19 \pm 2.48$ & \multirow[t]{2}{*}{$0.712^{*}$} \\
\hline Range & $(10-21)$ & $(10-18)$ & \\
\hline \multicolumn{4}{|l|}{ CMT } \\
\hline Mean \pm SD & $244.58 \pm 18.43$ & $248.42 \pm 19.27$ & \multirow[t]{2}{*}{$0.384 *$} \\
\hline Range & $(208-297)$ & $(210-289)$ & \\
\hline \multicolumn{4}{|l|}{ SFCT } \\
\hline Mean \pm SD & $274.47 \pm 35.88$ & $300.95 \pm 28.06$ & \multirow[t]{2}{*}{$0.001 *$} \\
\hline Range & $(199-334)$ & $(242-350)$ & \\
\hline
\end{tabular}

$D$ diopter, $I O P$ intraocular pressure, $S D$ standard deviation, $C M T$ central macular thickness, $S F C T$ subfoveal choroidal thickness

$* t$ test

**Pearson chi-square 
Table 2 Demographic and clinical characteristics of SSc subtypes

\begin{tabular}{lccc}
\hline & $\operatorname{DcSSc}(n=15)$ & $\operatorname{LcSSc}(n=30)$ & $p$ value \\
\hline Age (years) & $52.00 \pm 9.05$ & $52.00 \pm 11.79$ & $0.977^{*}$ \\
$\begin{array}{l}\text { Duration of SSc } \\
\text { (years) }\end{array}$ & $12.93 \pm 9.04$ & $9.30 \pm 7.61$ & $0.164^{*}$ \\
$\begin{array}{l}\text { Age at diagnosis } \\
\text { (years) }\end{array}$ & $38.14 \pm 10.74$ & $42.87 \pm 11.14$ & $0.192^{*}$ \\
$\begin{array}{l}\text { Anti-topo I positivity } \\
\text { (SCL70) (\%) }\end{array}$ & $60 \%$ & $43.3 \%$ & $0.292^{* *}$ \\
ACA positivity (\%) & $13.3 \%$ & $30 \%$ & $0.288^{* * *}$ \\
ANA positivity (\%) & $26.7 \%$ & $30 \%$ & $0.898^{* * *}$ \\
$\begin{array}{l}\text { Digital ulcers (\%) } \\
\text { DMARD (\%) (MTX, }\end{array}$ & $53.3 \%$ & $46.7 \%$ & $0.86^{* * *}$ \\
MMF, AZA, HCQ) & & $53.3 \%$ & $0.997^{* *}$ \\
\hline
\end{tabular}

$S S c$ systemic sclerosis, DcSSc diffuse cutaneous SSc, LcSSc limited cutaneous SSc, $A C A$ anti-centromere antibodies, anti-topo I anti-DNA topoisomerase I, ANA antinuclear antibody, DMARD diseasemodifying antirheumatic drugs

The value was statistically significant $(p<0.05)$

$* t$ test

**Pearson chi-square

***Fisher exact test

shows the clinical and demographic characteristics of the SSc patient and control groups.

Of these 45 patients, 15 (33.3\%) had a diagnosis of diffuse cutaneous SSc (DcSSc), and 30 (66.7\%) had a diagnosis of limited cutaneous SSc (LcSSc). Table 2 shows the clinical and serological profile in SSc subtypes. No significant differences were found in terms of age, age at diagnosis, duration of the disease, anti-topo I positivity, anti-centromere antibody (ACA) positivity, antinuclear antibody (ANA) positivity, rate of digital ulcers and disease-modifying drug (DMARD) use rates among the SSc subtypes, as shown in Table $2(p>0.05$ for all).

Table 3 shows OCTA variables for SSc and control eyes. No significant difference was found in FAZ assessment tool variables (FAZ area, FAZ perimeter, AI of the FAZ, foveal density-300) between SSc and healthy control eyes $(p>$ 0.05 for all). The SCP and DCP vessel densities are also shown in detail in Table 3 (Fig. 2). The SCP vessel densities of the whole image, fovea, parafovea and perifovea were significantly lower in patients with SSc than in healthy control subjects $(p=0.013, p=0.005, p=0.002, p=$ 0.018 , respectively). The DCP vessel densities of patients with SSc were not significantly different from those of healthy control subjects $(p>0.05$ for all), except for the fovea in which the DCP vessel density was significantly lower in SSc patients than in healthy control subjects $(34.61 \pm 6.38$ vs. $38.16 \pm 8.44, p=0.044)$. There was no significant difference in the choriocapillaris flow area between patients with SSc and healthy control subjects $(p>$ 0.05 for all).

OCTA nonflow, flow and FAZ assessment tool parameters were similar between the DcSSc and LcSSc subtypes of SSc patients $(p>0.05)$. There was no correlation between the duration of the disease and vessel densities in SCP or DCP in patients with SSc ( $p>0.05$ for all).

Table 4 shows the correlations between vessel densities and macular and choroidal thickness values in SSc patients. In patients with SSc, the foveal vessel density at SCP and the foveal vessel density at DCP correlated significantly with CMT $(p<0.001, p=0.002)$, and the foveal vessel density at DCP correlated significantly with SFCT $(p=$ 0.002). There was no statistically significant correlation of SFCT with choriocapillaris flow area; however, there was a significant correlation of SFCT with CMT ( $p=0.413, p=$ 0.034).

\section{Discussion}

The pathophysiology of SSc is characterized by immune activation, vasculopathy, and widespread fibrosis. SSc mainly targets small arteries and capillaries, and the disease is characterized by the obliteration of vessels and reduced capillary density [1-4]. The detection of the early involvement of ocular microcirculation is important because retinal and choroidal vasculature would be ideal for the observation of generalized arteriolar and capillary pathology in SSc. Although some studies have focused on the choroidal and retinal vascular characteristics in eyes with SSc, specific and comprehensive analyses at different depths of the retina and choroid using OCTA are still needed [9-11].

Some studies have shown evidence of subclinical retinal and choroidal vessel involvement in the early phase of SSc. Farkas's post-mortem histopathologic study identified basement membrane and endothelial cell abnormalities consisting of diffuse basement membrane thickening and endothelial swelling as well as necrosis as a basic defect in the choroidal vasculature in advanced SSc [6]. Gomes et al. revealed retinal microvascular abnormalities (28.9\%), including generalized and focal arteriolar narrowing, arteriovenous nicking, and vascular tortuosity. Additionally, Ushiyama et al. found a higher frequency (34\%) of retinal changes comprising hard exudates, vascular tortuosity, microhaemorrhage, and macular degeneration in SSc eyes compared to those in control eyes [7, 8]. In another study, Serup et al. demonstrated late phase angiographic changes $(33 \%)$, indicating that choroidal perfusion is affected in SSc patients with no evidence of concomitant arterial diseases and no history or other evidence of known ocular disease [12]. Both Serup and Grennan found angiographic alterations consisting of hyperfluorescence of the retinal pigment 
Table 3 OCTA comparison between eyes of SSc patients and controls

\begin{tabular}{|c|c|c|c|}
\hline Variable & $\begin{array}{l}\text { SSc } \\
\text { Mean } \pm \text { SD }\end{array}$ & $\begin{array}{l}\text { Control } \\
\text { Mean } \pm \text { SD }\end{array}$ & $p$ value \\
\hline $\mathrm{FAZ}$ area(mm2) (in $\mathrm{SCP}$ ) & $0.54 \pm 0.13(0.28-0.95)$ & $0.51 \pm 0.16(0.30-0.90)$ & 0.235 \\
\hline $\mathrm{FAZ}$ area(mm2) (in full retina) & $0.31 \pm 0.10(0.07-0.55)$ & $0.27 \pm 0.13(0.09-0.57)$ & 0.099 \\
\hline Perimeter (mm) & $2.12 \pm 0.35(1.12-2.85)$ & $1.95 \pm 0.48(1.18-2.90)$ & 0.051 \\
\hline Acircularity index & $1.10 \pm 0.03(1.04-1.20)$ & $1.09 \pm 0.02(1.05-1.13)$ & 0.612 \\
\hline Foveal density (\%) & $54.23 \pm 3.96(46.78-61.19)$ & $54.68 \pm 3.91(44.02-62.14)$ & 0.593 \\
\hline \multicolumn{4}{|l|}{ Vessel density SCP\% } \\
\hline Whole image & $49.79 \pm 3.47(42.60-56.30)$ & $51.49 \pm 2.89(43.10-56.50)$ & $0.013 *$ \\
\hline Superior-hemi & $49.80 \pm 3.65(41.70-56.60)$ & $51.30 \pm 2.84(43.20-55.80)$ & $\mathbf{0 . 0 3 3}^{*}$ \\
\hline Inferior-hemi & $49.78 \pm 3.40(42.30-56.10)$ & $51.66 \pm 3.08(42.90-57.20)$ & $0.007 *$ \\
\hline Fovea & $18.20 \pm 5.91(2.10-34.60)$ & $22.20 \pm 7.18(3.70-36.40)$ & $0.005 *$ \\
\hline Parafovea & $51.45 \pm 4.25(42.90-58.00)$ & $54.23 \pm 2.76(46.10-58.30)$ & $P<0.001$ * \\
\hline Temporal & $51.76 \pm 4.05(41.70-57.80)$ & $54.18 \pm 2.95(47.10-58.40)$ & $0.002 *$ \\
\hline Superior & $51.79 \pm 6.22(35.70-59.00)$ & $55.00 \pm 3.37(46.50-59.90)$ & $0.003 *$ \\
\hline Nasal & $50.64 \pm 3.97(39.60-58.30)$ & $53.26 \pm 3.05(47.10-60.00)$ & $p<0.001 *$ \\
\hline Inferior & $52.15 \pm 4.61(43.50-60.10)$ & $54.03 \pm 3.37(43.50-60.00)$ & $0.029 *$ \\
\hline Perifovea & $50.42 \pm 3.75(42.90-56.30)$ & $52.21 \pm 3.27(42.40-57.90)$ & $0.018 *$ \\
\hline Temporal & $46.54 \pm 3.68(37.20-57.50)$ & $48.86 \pm 3.63(38.20-57.90)$ & $0.010 *$ \\
\hline Superior & $50.44 \pm 4.20(40.70-57.10)$ & $52.18 \pm 4.20(40.70-67.10)$ & 0.052 \\
\hline Nasal & $54.34 \pm 3.43(44.40-61.20)$ & $56.01 \pm 3.18(48.00-61.40)$ & $0.019 *$ \\
\hline Inferior & $50.70 \pm 3.88(43.20-57.50)$ & $52.52 \pm 3.87(42.60-59.20)$ & $0.028 *$ \\
\hline \multicolumn{4}{|l|}{ Vessel density DCP \% } \\
\hline Whole image & $51.56 \pm 6.52(39.10-64.10)$ & $52.44 \pm 5.43(38.70-62.10)$ & 0.479 \\
\hline Superior-hemi & $52.00 \pm 6.85(39.70-64.10)$ & $52.98 \pm 5.74(37.50-61.00)$ & 0.446 \\
\hline Inferior-hemi & $50.91 \pm 8.00(36.80-64.20)$ & $51.85 \pm 5.29(20.80-48.50)$ & 0.467 \\
\hline Fovea & $34.61 \pm 6.38(15.50-47.90)$ & $38.16 \pm 8.44(39.90-63.40)$ & $0.044 *$ \\
\hline Parafovea & $55.57 \pm 4.55(46.10-63.00)$ & $55.87 \pm 4.05(43.00-62.00)$ & 0.744 \\
\hline Temporal & $56.06 \pm 4.80(47.70-64.00)$ & $56.58 \pm 3.09(50.40-61.30)$ & 0.398 \\
\hline Superior & $55.34 \pm 5.06(45.60-63.50)$ & $55.09 \pm 3.89(48.00-60.60)$ & 0.146 \\
\hline Nasal & $56.06 \pm 4.49(47.70-64.80)$ & $56.75 \pm 3.94(48.10-64.70)$ & 0.437 \\
\hline Inferior & $54.17 \pm 5.21(40.70-62.00)$ & $53.74 \pm 4.90(39.30-60.90)$ & 0.684 \\
\hline Perifovea & $52.68 \pm 7.12(38.00-65.60)$ & $54.13 \pm 5.78(38.50-64.20)$ & 0.294 \\
\hline Temporal & $54.73 \pm 6.29(43.00-66.10)$ & $56.52 \pm 4.72(44.20-64.50)$ & 0.130 \\
\hline Superior & $52.53 \pm 8.03(35.90-65.50)$ & $53.66 \pm 6.80(34.00-65.70)$ & 0.475 \\
\hline Nasal & $52.30 \pm 7.58(35.30-65.50)$ & $53.37 \pm 6.28(38.60-64.90)$ & 0.468 \\
\hline Inferior & $51.45 \pm 8.18(34.90-65.50)$ & $52.96 \pm 6.44(34.60-62.10)$ & 0.335 \\
\hline \multicolumn{4}{|c|}{ CCP choriocapillaris flow area $\mathrm{mm}^{2}$} \\
\hline $1 \mathrm{~mm}$ & $2.13 \pm 0.12(1.93-2.68)$ & $2.11 \pm 0.12(1.65-2.38)$ & 0.343 \\
\hline $2 \mathrm{~mm}$ & $8.70 \pm 0.30(8.03-9.33)$ & $8.63 \pm 0.43(7.16-9.63)$ & 0.396 \\
\hline $3 \mathrm{~mm}$ & $19.93 \pm 0.66(18.48-21.40)$ & $19.92 \pm 0.85(17.41-21.96)$ & 0.933 \\
\hline
\end{tabular}

All data are presented as the means $\pm \mathrm{SD}$

OCTA optical coherence tomography angiography, FAZ foveal avascular zone, SCP superficial capillary plexus, $D C P$ deep capillary plexus

*The value was statistically significant $(p<0.05)$ epithelium layer, most likely caused by a vascular lesion of the choroidal layer, but hyperfluorescence was uncommon for retinal arterioles [12, 13]. Waszczykowska et al. identified vascular abnormalities (55\%) with fluorescein angiography, such as delayed filling of choroidal lobules, occlusion of the peripheral arteries, thinning of retinal pigment epithelium and choroidal capillaries, ischemic areas surrounded by microaneurysms and intraretinal 

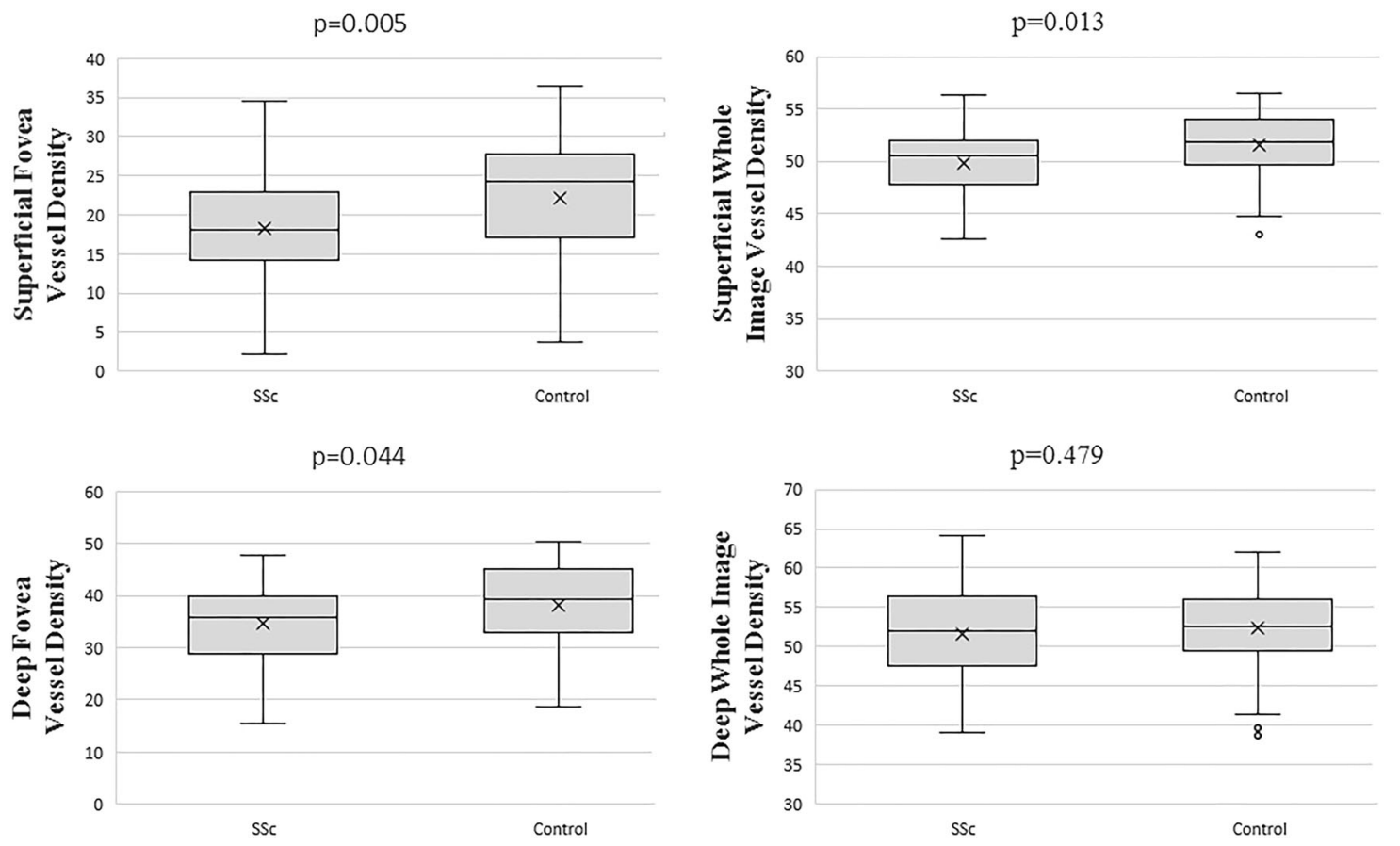

Fig. 2 Vessel densities of the retinal superficial retinal capillary plexus (SCP) and deep retinal capillary plexus (DCP). Significant differences were tested using $t$ tests. $P<0.05$ was considered statistically significant

Table 4 Correlations between vessel densities with macular and choroidal thickness values in SSc patients

\begin{tabular}{|c|c|c|}
\hline & $\begin{array}{l}\text { Central macular thickness }(\mu \mathrm{m}) \\
\text { (ILM-RPE) }\end{array}$ & $\begin{array}{l}\text { Subfoveal choroidal } \\
\text { thickness }(\mu \mathrm{m})\end{array}$ \\
\hline \multicolumn{3}{|l|}{$\operatorname{SCP}(\%)$} \\
\hline Whole image VD & $r=0.617, p=0.383$ & $r=-0.06, p=0.524$ \\
\hline Fovea VD & $\boldsymbol{r}=0.845, \boldsymbol{p}<\mathbf{0 . 0 0 1}$ & $r=0.226, p=0.186$ \\
\hline \multicolumn{3}{|l|}{ DCP $(\%)$} \\
\hline Whole image VD & $r=0.045, p=0.793$ & $r=0.073, p=0.672$ \\
\hline Fovea VD & $\boldsymbol{r}=0.506, \boldsymbol{p}=\mathbf{0 . 0 0 2}$ & $\boldsymbol{r}=0.507, \boldsymbol{p}=\mathbf{0 . 0 0 2}$ \\
\hline Choriocapillaris flow area $\left(\mathrm{mm}^{2}\right)$ & $r=0.104, p=0.548$ & $r=0.141, p=0.413$ \\
\hline
\end{tabular}

$r$ : Pearson correlation coefficient. $p<0.05$ statistically significant extravasation, capillary nonperfusion in the periphery and enhanced capillary network [14]. Minasion et al. reported bilateral ischemic retinopathy and neovascularisation at the disc in association with SSc [15]. Additionally, Milenkovic et al. reported choroidal sclerosis along with capillary nonperfusion in the retinal circulation in a middle-aged patient with localized scleroderma [16]. In Abdellatief's case report, choroidal vessel leakage in both eyes of a patient with SSc was determined with indocyanine green angiography [17]. Moreover, some studies have reported decreased choroidal thickness in patients with SSc without any evident eye disease [18-20].

In our study, we demonstrated differences between SSc eyes and healthy control eyes in terms of foveal capillary plexus vessel densities by using OCTA. Compared with control eyes, SSc eyes had significantly decreased foveal, parafoveal and perifoveal vessel densities at SCP and foveal vessel density at DCP. These reduced vessel densities were significantly correlated with CMT. On OCT, a thinned SFCT has been observed in SSc patients, and this finding is consistent with impaired choroidal perfusion. The choroidal circulation, with its dense monolayer of broad capillaries (choriocapillaris), differs in structure from the retinal circulation with its small capillary bed [21, 22]. In OCTA imaging, the choriocapillaris, with an overall thickness of $10 \mu \mathrm{m}$ at the subfoveal location, is the most clearly investigable choroidal tissue with OCTA; however, making assumptions regarding total choroidal perfusion based on this structure is limited to some extent [23]. With this limitation serving as an obstacle, no correlation was found between the SFCT and choriocapillaris flow area by using OCTA. 
Our study also revealed features of OCTA in SSc subtypes. In this study, we found no significant difference in mean OCTA measurements between LSSc and DSSc subtypes. The main difference between these two subtypes is the rate of disease progression and the extent and severity of skin and visceral involvement [24]. Patients with LcSSc have an insidious onset of skin sclerosis distal to their elbows and knees and have facial involvement. Patients with DcSSc develop proximal sclerosis, in addition to distal sclerosis. In DcSSc, disease progression is very fast with earlier and more widespread internal organ involvement. Patients with LcSSc are more likely to have ACA antibodies, whereas patients with DcSSc are more likely to have anti-topo I or anti-RNA polymerase III antibodies [3]. The prevalence of the positivity of the autoantibodies studied in this sample was $28.9 \%$ for ANA, $48.9 \%$ for anti-topo I and $24.4 \%$ for ACA. As expected, anti-topo I was more common in patients with the diffuse form of the disease, and ACA was more common in the limited form.

To the best of our knowledge, this study is the first to investigate retinal and choroidal microvascular alterations in SSc patients by using OCTA. In addition to the many strengths of this study, there are some limitations, such as the small sample size and cross-sectional nature. Moreover, we could not assess the vessel density of the choriocapillaris, which is the principal blood supply to the outer retina, with the XR Avanti AngioVue OCTA (version 2017.1.0.152). Therefore, longitudinal studies investigating microvascular changes over time in SSc patients are still needed.

In conclusion, we comprehensively illustrated the microvascular characteristics of patients with SSc without any clinical signs of retinopathy and found that SSc affects the vessel density of the inner retina, supporting the hypothesis of widespread vascular injury and microvascular changes in patients with SSc. These microvascular alterations observed in our study may be early indicators of retinal vascular injury long before patients become symptomatic.

\section{Summary}

\section{What was known before}

- Systemic sclerosis is a multisystemic connective tissue disease characterized by obliterative vasculopathy, which is not only limited to skin but may affect any internal organs. The prevalence of retinal vascular disease in systemic sclerosis is between 29 and 55\%, and retinal injury may occur long before the patients become symptomatic.

\section{What this study adds}

- This study demonstrated reduced vessel density of inner retina using optical coherence tomography angiography in patients with systemic sclerosis without any clinical signs of retinopathy. The ability of optical coherence tomography angiography to disclose early vascular alterations in patients with systemic sclerosis may play a significant role in the assessment of prognosis in the near future.

\section{Compliance with ethical standards}

Conflict of interest The authors declare that they have no conflict of interest.

Publisher's note Springer Nature remains neutral with regard to jurisdictional claims in published maps and institutional affiliations.

\section{References}

1. LeRoy E, Black C, Fleischmajer R, Jablonska S, Krieg T, Medsger TA Jr, et al. Scleroderma (systemic sclerosis): classification, subsets and pathogenesis. J Rheumatol. 1988;15:202-5.

2. Sakkas LI. New developments in the pathogenesis of systemic sclerosis. Autoimmunity. 2005;38:113-6.

3. Galluccio F, Walker U, Nihtyanova S, Moinzadeh P, Hunzelmann N, Krieg T, et al. Registries in systemic sclerosis: a worldwide experience. Rheumatol (Oxf). 2011;50:60-68.

4. Matucci-Cerinic M, Kahaleh B, Wigley FM. Review: evidence that systemic sclerosis is a vascular disease. Arthritis Rheum. 2013;65:1953-62.

5. Hekimsoy V, Kaya EB, Akdogan A, Sahiner L, Evranos B, Canpolat U, et al. Echocardiographic assessment of regional right ventricular systolic function using two-dimensional strain echocardiography and evaluation of the predictive ability of longitudinal 2D-strain imaging for pulmonary arterial hypertension in systemic sclerosis patients. Int J Cardiovasc Imaging. 2018;34: 883-92.

6. Farkas TG, Sylvester V, Archer D. The choroidopathy of progressive systemic sclerosis (scleroderma). Am J Ophthalmol. 1972;74:875-86.

7. Gomes Bde A, Santhiago MR, Magalhaes P, Kara-Junior N, Azevedo MN, Moraes HV, et al. Ocular findings in patients with systemic sclerosis. Clinics. 2011;66:379-85.

8. Ushiyama O, Ushiyama K, Yamada T, Koarada S, Tada Y, Suzuki $\mathrm{N}$, et al. Retinal findings in systemic sclerosis: a comparison with nailfold capillaroscopic patterns. Ann Rheum Dis. 2003;62:204-7.

9. Savastano MC, Lumbroso B, Rispoli M. In vivo characterization of retinal vascularization morphology using optical coherence tomography angiography. Retina. 2015;35:2196-203.

10. Chen H, Chi W, Cai X, Deng Y, Jiang X, Wei Y, et al. Macular microvasculature features before and after vitrectomy in idiopathic macular epiretinal membrane: an OCT angiography analysis. Eye (Lond). 2019;33:619-28.

11. Mo S, Krawitz B, Efstathiadis E, Geyman L, Weitz R, Chui TY, et al. Imaging foveal microvasculature: optical coherence tomography angiography versus adaptive optics scanning light 
ophthalmoscope fluorescein angiography. Invest Ophthalmol Vis Sci. 2016;57:130-40.

12. Serup L, Serup J, Hagdrup H. Fundus fluorescein angiography in generalized scleroderma. Ophthalmic Res. 1987;19:303-8.

13. Grennan DM, Forrester J. Involvement of the eye in SLE and scleroderma. A study using fluorescein angiography in addition to clinical ophthalmic assessment. Ann Rheum Dis. 1977;36:152-6.

14. Waszczykowska A, Goś R, Waszczykowska E, DziankowskaBartkowiak B, Piotr Jurowski P. Prevalence of ocular manifestations in systemic sclerosis patients. Arch Med Sci. 2013;9: 1107-13.

15. Minasion M, Stanford M, Graham E, Denton CP, Black C. Bilateral ischaemic retinal vasculopathy in scleroderma. $\mathrm{Br} \mathrm{J}$ Ophthalmol. 2005;89:1063-71.

16. Milenkovic S, Petrovic L, Risimic D, Kosanovic-Jakovic N, Jaksic V, Djakovic Z, et al. Choroidal sclerosis in localized scleroderma (morphea en plaque). Ophthalmic Res. 2008;40: $101-4$.

17. Abdellatief A, Balasubramaniam SC, Grube TJ, Gonzalez Santiago TM, Osborn TG, Pulido JS. Indocyanine green angiographic evidence of choroiditis in scleroderma. Retin Cases Brief Rep. 2015;9:231-4.
18. Ingegnoli F, Gualtierotti R, Pierro L, Del Turco C, Miserocchi E, Schioppo T, et al. Choroidal impairment and macular thinning in patients with systemic sclerosis: the acute study. Microvasc Res. 2015;97:31-36.

19. Coşkun E, Zengin O, Kenan S, Kimyon G, Erdogan Er K, Okumus $\mathrm{S}$, et al. Evaluation of choroidal thickness in patients with scleroderma. Eye. 2016;30:588-92.

20. Esen E, Tas DA, Sizmaz S, Turk I, Unal I, Demircan N. Evaluating choroidal characteristics in systemic sclerosis using enhanced depth imaging optical coherence tomography. Ocul Immunol Inflamm. 2017;25:356-62.

21. Delaey C, Van De, Voorde J. Regulatory mechanisms in the retinal and choroidal circulation. Ophthalmic Res. 2000;32:249-56.

22. Chirco KR, Sohn EH, Stone EM, Tucker BA, Mullins RF. Structural and molecular changes in the aging choroid: implications for age-related macular degeneration. Eye. 2017;31:10-25.

23. Gorczynska I, Migacz JV, Zawadzki RJ, Capps AG, Werner JS. Comparison of amplitude-decorrelation, speckle-variance and phase-variance OCT angiography methods for imaging the human retina and choroid. Biomed Opt Express. 2016;7:911-42.

24. Krieg T, Takehara K. Skin disease: a cardinal feature of systemic sclerosis. Rheumatol (Oxf). 2009;48(Suppl 3):iii14-iii18. 